\title{
Redes Complexas e Sociais como ferramentas para o estudo de um sistema complexo baseado em keywords de produções científicas
}

\author{
Nascimento, J. O. do ${ }^{1 *}$; Pereira, H. B. $B^{1,2}$; Moret, M. $A^{1,2}$ \\ 1 Programa de Modelagem Computacional, CIMATEC, Salvador, BA, Brasil. \\ 2 Universidade do Estado da Bahia, Salvador, Bahia, Brasil.
}

*e-mail: jeffersonascimento@gmail.com

\begin{abstract}
Resumo
Dentre as diversas características pertencentes aos sistemas complexos, destacamos as interações entre as várias entidades que o constituem, ocorrendo então a emergência de propriedades que resultam no comportamento do todo. Quando temos um texto, as entidades correspondem as unidades significantes ou palavras, e as suas interações ocorrem por meio das sentenças do texto. Este artigo tem como objetivo apresentar um sistema complexo verificado por meio da Teoria de Redes, através de redes semânticas baseadas em palavras-chave, que analisa a evolução do Ensino de Física nacional entre os períodos de 1992-2006. Apresentamos o método de construção das redes semânticas observando as nuances de pré-processamento dos dados e a modelagem computacional propriamente dita com a utilização de softwares para construção e visualização das redes. Os índices estatísticos pertencentes a teoria de redes complexas e as medidas de centralidades de grau e proximidade pertencentes às redes sociais são calculados e apresentados (ambos para redes estáticas no tempo). Pelos padrões apresentados, as redes semânticas topologicamente, caracterizam-se apresentando o fenômeno SmallWorld e há indicativos de que também sejam redes Scale Free.
\end{abstract}

Palavras-chave: Sistemas Complexos, Redes Sociais e Complexas, Redes Semânticas e Ensino de Física

\begin{abstract}
Among the several characteristics belonging to the complex systems, we highlight the interactions between the various entities that constitute it, occurring then the emergence of properties that result in the behavior of the whole. When we have a text, the entities correspond to the signifying units or words, and their interactions occur through the sentences of the text. This article aims to present a complex system verified through the Network Theory, through semantic networks based on keywords, that analyzes the evolution of the National Physics Teaching between the periods of 1992-2006. We present the method of construction of the semantic networks observing the nuances of data preprocessing and the computational modeling itself with the use of software for the construction and visualization of the networks. The statistical indices belonging to complex networks theory and the measures of centralities of degree and proximity belonging to social networks are calculated and presented (both for static networks in time). By the presented standards, the semantic networks topologically, characterize presenting the Small-World phenomenon and there are indicatives that they are also Scale Free networks.
\end{abstract}

Keywords: Complex Systems, Social and Complex Networks, Semantic Networks and Physics Teaching

\section{Introdução}

Algumas décadas atrás as diversas áreas científicas possuíam técnicas e características próprias para estudos e análises dos padrões estudados em seus sistemas [1]. Nas palavras da autora este pensamento abrangia das ciências físicas à antropologia, assim como, da economia à biologia. Desde o início dessas pesquisas, em sistemas 
complexos, apontaram à direção que todos esses sistemas congregavam propriedades universais [1,2]. A investigação dos sistemas complexos ocorre por meio do formalismo matemático de sistemas dinâmicos, através de equações diferenciais, equações por meio de diferenças, mapas logísticos, autômatos celulares, redes dinâmicas (teoria de redes), etc. [1,2], cuja ferramenta fundamental é a utilização de recursos computacionais [3]. A teoria de Redes Complexas e Redes sociais correspondem a formas de estudarmos sistemas complexos [3].

Inspirados em Nascimento et al. [3] e Nascimento, Pereira e Moret [4], a presente pesquisa tem como objetivo apresentar um sistema complexo, verificado por meio da Teoria de Redes, através de redes semânticas baseadas em Keywords (palavraschave) de produções científicas. As análises realizadas são aplicadas, na temática do Ensino de Física nacional, entre os períodos de 1992-2006

Este artigo está organizado em 5 seções. $\mathrm{Na}$ próxima, apresentaremos os materiais e métodos que utilizamos para construção da rede semântica. $\mathrm{Na}$ terceira, os resultados encontrados por meio das análises das redes semânticas. Na quarta seção, as considerações finais do presente artigo. Na última sessão, as referências utilizadas na elaboração deste artigo. .

\section{Materiais e Métodos}

A base de dados para a construção e análise das redes semânticas são os disponibilizados em Salem e Kawamura [5,6]. Conforme a Teoria de Redes, para representarmos uma determinada rede semântica, utilizaremos os princípios pertencentes à Teoria dos Grafos. Sendo assim, um grafo $G=(V, E)$ consiste em uma estrutura matemática composta por dois conjuntos: o conjunto de vértices $(\mathrm{V})$, que é finito e não vazio) e, o conjunto das arestas $(E)$, as relações binárias sobre V) [7]. Os índices estatísticos pertencentes à Teoria dos Grafos utilizados utilizamos na observância das redes semânticas, da presente pesquisa são:

Tabela 1: índices pertencentes às redes complexas. Fonte: Pereira et al. [8] e Nascimento et. al. [3].

\begin{tabular}{c|c|c}
\hline$n$ & Número de Vértices & $n=|V|$ \\
\hline$m$ & Número de Arestas & $m=|E|$ \\
\hline$\langle k\rangle$ & Grau Médio do Vértice & $\langle k\rangle=\frac{1}{n} \sum_{i}^{n} k_{i}$ \\
\hline$\Delta$ & Densidade da Rede & $\Delta=\frac{m}{n(n-1) / 2}$ \\
\hline$\left(C_{v}\right)$, & Coeficiente de & $C_{V}=\frac{2 E_{v}}{k_{v}\left(k_{v}-1\right)}$ \\
\hline$\left.C_{w s}\right)$ & Aglomeração do Vértice & $C_{W S}=\frac{1}{N} \sum_{V=1}^{N} C_{V}$ \\
\hline$L$ & Caglomeração da Rede & \multicolumn{1}{c}{${ }^{\prime}$} \\
\hline$D$ & Diâmetro da Rede & $D=\frac{\max \left(d_{i j}\right)}{n(n-1)} \sum_{i \neq j}$ \\
\hline
\end{tabular}

Em relação ao método de construção da rede semântica complexa baseada em keywords (palavraschave), desde o pré-processamento das palavras até a utilização de softwares, estão apresentados nas obras de Pereira et al. [8], Nascimento et al. [2], Nascimento, Pereira e Moret [4] e Nascimento et al. [9].

\section{Resultados}

De acordo com as informações disponíveis na sessão anterior, apresentamos na Figura 1 a rede semântica complexa baseada em palavras-chave de dissertações e teses em Física (Ensino) no período de 1992-1995:

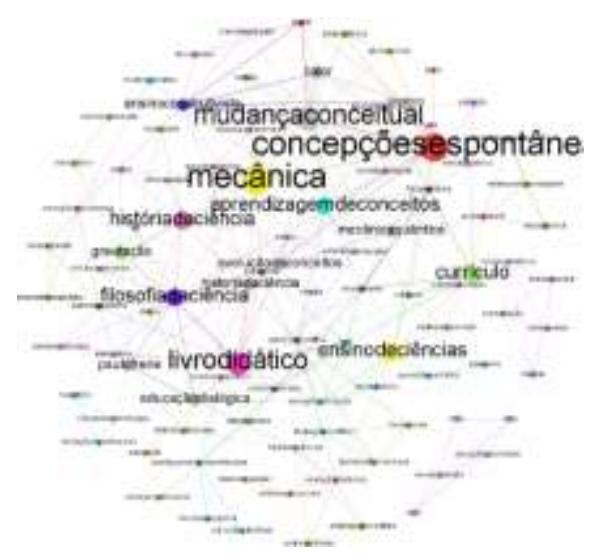

Figura 1: Rede semântica baseada em palavras-chave (1992-1995).

A rede semântica da Figura 1 apresenta os tamanhos dos vértices e os seus respectivos nomes proporcionais em tamanho, aos valores medidos da centralidade de grau (medida de importância de um 
vértice por meio das conexões com vértices vizinhos). $\mathrm{Na}$ Tabela 2 estão disponíveis os índices de redes complexas conforme a Tabela 1, verificados para a rede semântica da Figura 1:

Tabela 1: índices pertencentes às redes complexas.

\begin{tabular}{|c|c|c|c|c|}
\hline Rede & Îndices & Valor & Î́ndices & Valor \\
\hline \multirow[t]{4}{*}{ Keywords } & $n=|V|$ & 97 & $\begin{array}{c}\text { Quantidade } \\
\text { de } \\
\text { componentes }\end{array}$ & 4 \\
\hline & $m=|E|$ & 276 & $\langle k\rangle$ & 5,691 \\
\hline & $\Delta$ & 0,059 & $c_{w s}$ & 0,743 \\
\hline & $L$ & 3,036 & $D$ & 6 \\
\hline \multirow{4}{*}{$\begin{array}{c}\text { Keywords } \\
\text { (Maior } \\
\text { Componente) }\end{array}$} & $n=|V|$ & 85 & $\begin{array}{c}\text { Maior } \\
\text { Componente } \\
(\%)\end{array}$ & 87,63 \\
\hline & $m=|E|$ & 255 & $\langle k\rangle$ & 6 \\
\hline & $\Delta$ & 0,071 & $C_{w s}$ & 0,711 \\
\hline & $L$ & 3,048 & $D$ & 6 \\
\hline \multirow{4}{*}{$\begin{array}{c}\text { Rede } \\
\text { aleatória } \\
\text { equivalente. }\end{array}$} & $n=|V|$ & 85 & $\begin{array}{c}\text { Maior } \\
\text { Componente } \\
(\%)\end{array}$ & 100 \\
\hline & $m=|E|$ & 226 & $\langle\boldsymbol{k}\rangle$ & 6 \\
\hline & $\Delta$ & 0.073 & $C_{W S}$ & 0.082 \\
\hline & $L$ & 2.6053 & $D$ & 5 \\
\hline
\end{tabular}

A rede semântica baseado em palavras-chave para o período de 1996-2006, bem como seus respectivos índices estão presentes nas pesquisas de Nascimento, Pereira e Moret [4] e Nascimento et al. [9]. A rede semântica da Figura 2 é a correspondente ao período total, 1992-2006 e, apresenta os vértices com maiores centralidades de grau em seu centro (alguns estão destacados e nomeados). Os vértices mais distantes do centro, correspondem aos que apresentam os menores valores de centralidade de grau.

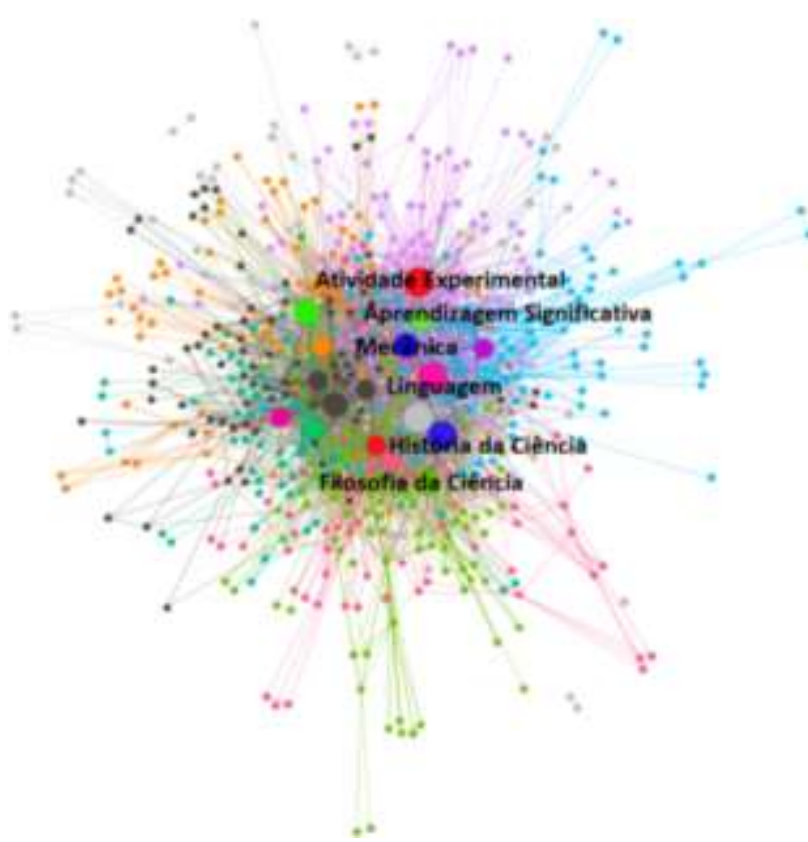

Figura 2: Rede semântica baseada em palavras-chave de dissertações e teses em Física (ensino).

A rede semântica da Figura 1 foi construída de modo a apresentar em seu centro os vértices (palavraschave) com maiores números de conexões na rede. Alguns dos vértices mais conectados na rede, estão presentes na Figura 1. Os vértices mais conectados na rede apresentam maiores importâncias (medida de centralidade de grau, pertencente às redes sociais). $\mathrm{Na}$ Tabela 2 estão disponíveis os índices de redes complexas apresentados na Tabela 1, verificados para a rede semântica da Figura 2:

Tabela 2: índices pertencentes às redes complexas.

\begin{tabular}{|c|c|c|c|c|}
\hline Rede & Índices & Valor & Índices & Valor \\
\hline \multirow{4}{*}{ Keywords } & $n=|V|$ & 664 & $\begin{array}{c}\text { Quantidade } \\
\text { de } \\
\text { componentes }\end{array}$ & 4 \\
\hline & $m=|E|$ & 2931 & $\langle k\rangle$ & 8,828 \\
\hline & $\Delta$ & 0,013 & $c_{w s}$ & 0,723 \\
\hline & $L$ & 3,048 & $D$ & 6 \\
\hline \multirow{4}{*}{$\begin{array}{c}\text { Keywords } \\
\text { (Maior } \\
\text { Componente) }\end{array}$} & $n=|V|$ & 657 & $\begin{array}{c}\text { Maior } \\
\text { Componente } \\
(\%)\end{array}$ & 98,95 \\
\hline & $m=|E|$ & 2926 & $\langle k\rangle$ & 8,907 \\
\hline & $\Delta$ & 0,014 & $C_{W s}$ & 0,722 \\
\hline & $L$ & 3,048 & $D$ & 6 \\
\hline \multirow{4}{*}{$\begin{array}{c}\text { Rede } \\
\text { aleatória } \\
\text { equivalente. }\end{array}$} & $n=|V|$ & 657 & $\begin{array}{c}\text { Maior } \\
\text { Componente } \\
(\%)\end{array}$ & 100 \\
\hline & $\boldsymbol{m}=|E|$ & 2966 & $\langle\boldsymbol{k}\rangle$ & 8,907 \\
\hline & $\Delta$ & 0.013 & $C_{w s}$ & 0,016 \\
\hline & $L$ & 3,189 & $D$ & 5 \\
\hline
\end{tabular}


Seguindo os nortes fornecidos por Watts e Strogatz [10], realizamos uma comparação entre as redes semânticas baseadas em palavras-chave (componente gigante) e as suas redes aleatórias equivalentes (uma rede com o mesmo número de vértice e grau médio). Observando a Tabela 1, a Tabela 2 e a comparação similar realizada em Nascimento, Pereira e Moret [4] percebemos que as redes apresentaram valores próximos para o caminho mínimo médio (L). Os coeficientes de aglomerações médios $\left(\mathrm{C}_{\mathrm{ws}}\right)$ das redes semânticas apresentaram valor elevado em comparação com o coeficiente de aglomeração da rede aleatória equivalente. Desta forma, as redes semânticas apresentam o fenômeno Small-Word: elevada aglomeração local e baixas geodésicas. A distribuição de graus das redes semânticas da Figura 2 e Figura 3 são os apresentados a seguir:

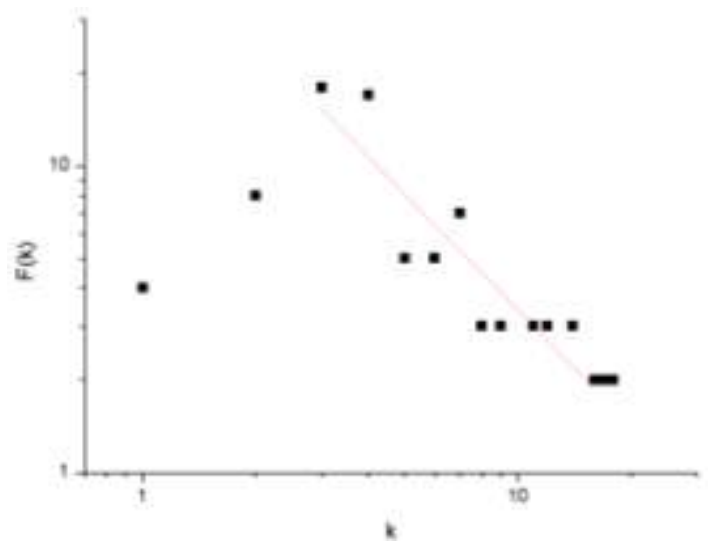

Figura 3: Distribuição de graus da rede semântica baseada palavraschave no período de 1992-1995 ( $\operatorname{com} \gamma=1,25214$ e o ajuste $R^{2}=$ 0,8045).

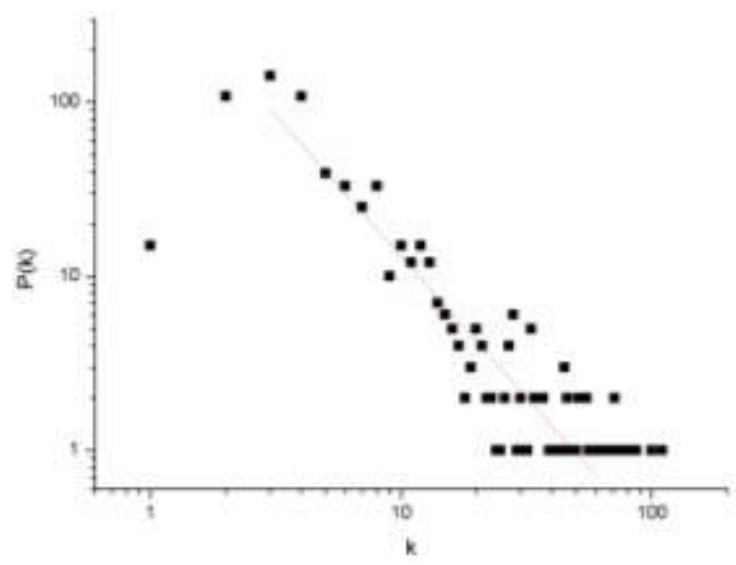

Figura 4: Distribuição de graus da rede semântica baseada em resumos ( $\operatorname{com} \gamma=1,619$ e o ajuste $\left.\mathbf{R}^{2}=0,83504\right)$.
Conforme as Figura 3 e Figura 4, a topologia das redes semânticas, sugerem seguir uma lei de potência da forma $F(k)=P(k) \sim k^{-\gamma}$, características de redes Scale Free (rede livre de escala) [11]. Uma rede pode apresentar o fenômeno Small-World e ser ScaleFree concomitantemente. Ambos as características são possíveis, pois, estas redes não apresentam características de exclusão entre si. A presença de hubs ocorre nessas redes e, elencamos os vinte primeiros vértices mais conectados na rede semântica da Figura 2:

Tabela 3: Centralidade de Grau dos vinte vértices (hubs) mais conectados na rede semântica (1992-2006).

\begin{tabular}{|c|c|c|c|c|c|}
\hline \multicolumn{2}{|r|}{$\begin{array}{l}\text { Vértices } \\
\text { (V) }\end{array}$} & \multirow{2}{*}{$\begin{array}{c}\begin{array}{c}\text { Grau } \\
(k)\end{array} \\
110\end{array}$} & \multicolumn{2}{|r|}{$\begin{array}{l}\text { Vértices } \\
\text { (V) }\end{array}$} & \multirow{2}{*}{$\begin{array}{c}\text { Grau } \\
(k) \\
59\end{array}$} \\
\hline 1 & $\begin{array}{c}\text { História da } \\
\text { Ciência }\end{array}$ & & 11 & $\begin{array}{l}\text { Concepção do } \\
\text { professor }\end{array}$ & \\
\hline 2 & $\begin{array}{l}\text { Livro } \\
\text { didático }\end{array}$ & 100 & 12 & $\begin{array}{c}\text { Aprendizagem } \\
\text { Significativa }\end{array}$ & 56 \\
\hline 3 & \begin{tabular}{|c|} 
Atividade \\
Experimental
\end{tabular} & 87 & 13 & $\begin{array}{c}\text { Formação inicial de } \\
\text { professores }\end{array}$ & 55 \\
\hline 4 & Linguagem & 81 & 14 & Currículo & 55 \\
\hline 5 & $\begin{array}{c}\text { Formação } \\
\text { continuada } \\
\text { de } \\
\text { professores }\end{array}$ & 75 & 15 & $\begin{array}{c}\text { Física moderna e } \\
\text { contemporânea }\end{array}$ & 51 \\
\hline 6 & $\begin{array}{c}\text { Formação } \\
\text { de } \\
\text { professores }\end{array}$ & 71 & 16 & $\begin{array}{l}\text { Concepções } \\
\text { espontâneas }\end{array}$ & 51 \\
\hline 7 & Mecânica & 71 & 17 & $\begin{array}{c}\text { Concepção do } \\
\text { aluno }\end{array}$ & 50 \\
\hline 8 & $\begin{array}{c}\text { Filosofia da } \\
\text { Ciência }\end{array}$ & 70 & 18 & Epistemologia & 48 \\
\hline 9 & $\begin{array}{c}\text { Proposta } \\
\text { didática }\end{array}$ & 69 & 19 & Mudança conceitual & 47 \\
\hline 10 & $\begin{array}{l}\text { Prática } \\
\text { docente }\end{array}$ & 64 & 20 & Interdisciplinaridade & 46 \\
\hline
\end{tabular}

Conforme os dados elencados na Tabela 3, há indícios que a rede semântica baseada em palavraschave para o período de 1992-2006, apresentou uma tendência, pelos seus hubs que objetivaram: a formação do professor de física; teorias de aprendizagens, com o destaque para a Teoria de David Ausubel; o foco de pesquisas voltadas à educação básica e uma forte presença de trabalhos desenvolvidos relacionadas com a história e filosofia das ciências. 


\section{Considerações finais}

A presente pesquisa apontou um sistema complexo representando por redes semânticas complexas e sociais presentes baseadas em palavraschave de dissertações e teses em Física (ensino). Os resultados indicaram que as redes apresentam 0 fenômeno Small-World e sugerem ser redes Scale Free. $O$ coeficiente angular $\gamma$ da lei de potência sugerido por Barabási e Albert [11] é $\gamma \geq 2,1$ e $\gamma \leq 4$. Porém, redes Scale Free com $\gamma$ menor do que 0 sugerido é recorrentemente encontrado em obras científicas, como Fadigas et al. [12], Seyed-allaei et al. [13] e Nascimento et al. [9].

Em relação a temática do Ensino de Física nacional as redes semânticas apresentaram algumas tendências. A formação de professores, metodologias de ensino e a educação básica foram alguns dos destaques presentes nas redes.

\section{Agradecimentos}

Jefferson Nascimento agradece à FAPESB por meio do apoio financeiro parcial recebido através da bolsa de doutorado (BOL170/2015). Marcelo A. Moret agradece ao $\mathrm{CNPq}$ pelo suporte financeiro parcial oriundo de sua bolsa de Produtividade em Pesquisa (No. 304454/2014-1).

\section{Referências}

[1] TEIXEIRA, Gesiane M. Redes Semânticas em discursos orais: Uma proposta metodológica baseada na psicologia cognitiva utilizando redes complexas. 2007. 118 f. Dissertação (Mestrado Interdisciplinar em Modelagem Computacional), - Centro de Pós graduação e Pesquisa da Fundação Visconde de Cairu. Fundação Visconde de Cairu, Salvador, 2007.

[2] CUNHA, M.V. Redes semânticas baseadas em títulos de artigos científicos. 127f. Dissertação (Mestrado em modelagem computacional e tecnologia industrial) Faculdade de Tecnologia Senai CIMATEC, Salvador, 27 nov. 2013.
[3] NASCIMENTO, J. O. do; PEREIRA-GUIZZO, C. S.; MOREIRA, D. M.; MONTEIRO, R. L. S.; PEREIRA, H. B. B.; MORET, M. A.; "Redes Sociais e Complexas: um modelo computacional para a investigação da pósgraduação Brasileira em Ensino de Física", p. 110-114 . In: Anais do VII Encontro Científico de Física Aplicada [=Blucher Physics Proceedings, v.3 n.1]. São Paulo: Blucher, 2016. ISSN 2358-2359, DOI 10.5151/phypro-vii-efa-027.

[4] NASCIMENTO, J. O. do.; PEREIRA, H. B. B.; MORET, M. A; "Redes semânticas baseadas em palavras-chave do Ensino de Física Brasileiro: uma comparação nos métodos de pré-processamento dos dados", p. 122-127 . In: . São Paulo: Blucher, 2017. ISSN 2358-2359, DOI 10.5151/phypro-viii-efa-28.

[5] SALEM, S.; KAWAMURA, M. R. D. Ensino de Física no Brasil: catálogo analítico de dissertações e teses (19721992). São Paulo: Instituto de Física da USP / PROFIS, 1992. $243 \mathrm{p}$.

[6] SALEM, S.; KAWAMURA, M. R. D. Ensino de Física no Brasil: catálogo analítico de dissertações e teses (19921995) São Paulo: s.n., 1996

[7] GROSS, J. L.; YELLEN, J. Graph theory and its applications. CRC press, Boca Raton FL USA 2005.

[8] PEREIRA, H. B. B. et al. Density: A measure of the diversity of concepts addressed in semantic networks. Physica A: Statistical Mechanics and its Applications, v. 441, p. 81-84, 2016.

[9] NASCIMENTO, J. O. do; MONTEIRO, R. L. S.; MOREIRA, D. M.; MORET, Marcelo A.; PEREIRA, H. B. B. Semantic networks of keywords from Brazilian dissertations on physics teaching. Discontinuity, Nonlinearity and Complexity, 2018.

[10] WATTS, Duncan J.; STROGATZ, Steven H. Collective dynamics of 'small-world'networks. Nature, v. 393, n. 6684, p. 440-442, 1998.

[11] BARABÁSI, A. L. E ALBERT, R.. "Emergence of scaling in random networks". Science, n. 286, pp. 509-512, 1999.

[12] FADIGAS, I. S., CASAS, T. H. P., SENNA, V., MORET, M. A, PEREIRA, H. B. B. "Análise de redes semânticas baseada em títulos de artigos de periódicos científicos: o caso dos periódicos de divulgação em educação matemática". Educação Matemática Pesquisa, 11, p. 167-193, 2009.

[13] SEYED-ALLAEI, H, BIANCONI \& M, G. MARSILI. Scale-free networks with an exponent less than two. Phys. Rev. E 73, 046113, 2006. 